\title{
Profiles in understanding operations with rational numbers
}

\author{
${ }^{1}$ González-Forte, J. M., ${ }^{1}$ Fernández, C., ${ }^{2}$ Van Hoof, J., and ${ }^{2}$ Van Dooren, W. \\ ${ }^{1}$ University of Alicante (Spain) \\ ${ }^{2}$ University of Leuven (Belgium)
}

\begin{abstract}
Students often show difficulties in understanding rational numbers. Often, these are related to the natural number bias, that is, the tendency to apply the properties of natural numbers to rational number tasks. Although this phenomenon has received a lot of research interest over the last two decades, research on the existence of qualitatively different profiles regarding students' understanding is scarce. The current study investigated the different ways students reasoned in arithmetic operation items with fractions and decimals. A cross-sectional study with 1,262 participants from $5^{\text {th }}$ to $10^{\text {th }}$ grade was performed. A TwoStep Cluster Analysis revealed eight different student reasoning profiles. We found that the natural number bias is first overcome in addition and subtraction, and later in multiplication and division. Moreover, differences regarding representation were only found in addition and subtraction items, indicating that natural numbers interfered more strongly in fractions than in decimal numbers. Finally, results showed that some students' difficulties with rational number multiplications and divisions had other explanations than the natural number bias.
\end{abstract}

Keywords: Rational numbers; fractions; decimal numbers; natural number bias; arithmetic operations, profiles.

\section{Introduction}

Rational numbers underlie the understanding of a wide range of more advanced mathematical concepts of algebra and calculus (Kieren, 1993). Moreover, the rational number is one of the most complex mathematical concepts in primary and secondary school (Behr, Lesh, Post, \& Silver, 1983). Understanding the rational number, its representations and its properties is difficult, and primary as well as secondary school students, and even undergraduates, have difficulties in understanding some of its aspects 
(Behr, Wachsmuth, Post, \& Lesh, 1984; Cramer, Post, \& delMas, 2002; Kerslake, 1986; Merenluoto \& Lehtinen, 2002; Vamvakoussi, Van Dooren, \& Verschaffel, 2012). These difficulties have been reflected in numerous and recurring errors as students order rational numbers, when they operate with them and with their representations (Fischbein, Deri, Nello, \& Marino, 1985; González-Forte, Fernández, Van Hoof, \& Van Dooren, 2020; Ni \& Zhou, 2005; Resnick et al., 1989; Stafylidou \& Vosniadou, 2004). Previous research has found that several of students' difficulties with rational numbers may be due to the extensive experience they have previously acquired with natural numbers and the ideas they have consequently developed about numbers more generally (McMullen, Laakkonen, Hannula-Sormunen, \& Lehtinen, 2015; Merenluoto \& Lehtinen, 2002; Namkung, Fuchs, \& Koziol, 2018; Resnick et al., 1989; Vamvakoussi et al., 2012). Rational number knowledge is not a simple extension of this natural number knowledge (Kieren, 1993). During the first years of rational number instruction, some students assume, implicitly or explicitly, that natural number rules and characteristics can be also applied to rational numbers (Van Dooren, Lehtinen, \& Verschaffel, 2015). This leads to difficulties as students find themselves in situations in which the principles and rules of natural numbers cannot be applied, leading to systematic errors when solving rational number tasks (Moss, 2005; Ni \& Zhou, 2005; Smith, Salomon, \& Carey, 2005; Vamvakoussi \& Vosniadou, 2004).

Based on this assumption, research has shown how primary and secondary school students, and even undergraduates, perform rational number tasks that are compatible with natural number knowledge (called congruent items in the literature) more accurately than tasks in which such knowledge is incompatible (incongruent items) (see for example: Van Hoof, Verschaffel, \& Van Dooren, 2015b). For instance, primary school students accurately determine that 0.67 is larger than 0.5 (the largest 
number has the longest natural number after the decimal point- congruent item), but they have difficulties in determining that 0.7 is larger than 0.35 (the largest number has the shortest natural number after the decimal point- incongruent item) (Moss, 2005). The phenomenon has been termed natural number bias (NNB) and is studied in three main domains where the properties of rational numbers differ from the properties of natural numbers: rational number size, density, and operations (Gómez \& Dartnell, 2019; McMullen et al., 2015; Obersteiner, Van Hoof, Verschaffel, \& Van Dooren, 2016; Vamvakoussi, Christou, \& Vosniadou, 2018; Van Hoof, Degrande, Ceulemans, Verschaffel, \& Van Dooren, 2018).

In the present study, we focus on the domain of arithmetic operations, both with fractions and decimal numbers. In the following section, we present a more extensive review of the literature in this field.

\section{Natural number bias in the domain of arithmetic operations}

This section is divided into three subsections. First, we review previous findings on students' difficulties regarding additions and subtractions with rational numbers. Second, we describe findings on difficulties in multiplications and divisions. Finally, we formulate the objective of the present study and discuss its contributions to the literature.

\section{Addition and subtraction}

Concerning additions and subtractions, some primary and secondary school students operate with fractions in the same way as they do with natural numbers, adding (or subtracting) numerators and denominators separately, without taking into account the relation between numerator and denominator (Moss, 2005; Streefland, 1991). An example of this incorrect assumption is considering that the result of the addition $3 / 4+$ 
$5 / 6$ is $8 / 10$, or the result of the subtraction $1 / 4-1 / 2$ is $0 / 2$ (Post, 1981; Siegler \& Pyke, 2013; Siegler, Thompson, \& Schneider, 2011; Streefland, 1991).

Understanding decimal number arithmetic does not require mastering as many different procedures as in the case of fractional arithmetic, but it does pose difficulties beyond the arithmetic of natural numbers (Lortie-Forgues, Tian, \& Siegler, 2015), namely because decimal arithmetic requires the correct placement of the decimal point. However, students often have difficulties when, for both addition and subtraction, the numbers involved do not have the same number of digits after the decimal point (Hiebert \& Wearne, 1985). In this way, they tend to add (subtract) the decimal part as if it were an addition (subtraction) of natural numbers. For instance, they consider that the result of the addition $0.19+0.2$ is 0.21 , or that the result of the subtraction $1.788-0.8$ is 1.780. In fact, previous research has indicated that primary and secondary school students achieve greater accuracy in additions and subtractions when the numbers involved had the same number of digits in the decimal part (e.g. $4.6+2.3$ and 0.60 0.36), than in those in which the number of digits was different (e.g. $5.3+2.42$ and 0.86

- 0.3) (Hiebert \& Wearne, 1985; Lortie-Forgues et al., 2015; Van Hoof, Vandewalle, Verschaffel, \& Van Dooren, 2015a).

\section{Multiplication and division}

Also in the domain of multiplication and division, some learners have been found to apply natural number properties to rational numbers (Fischbein et al., 1985). One of the widespread models associated with multiplication is the model of repeated addition in which the multiplier is the number of equivalent collections and the multiplicand is the size of each collection. This model could lead students to the intuitive rule that the product of a multiplication must always be a larger number than the factors (Bell, Swan, \& Taylor, 1981; Fischbein et al., 1985). In division, some students follow the intuitive 
model of a partitive division that implies sharing into $n$ equal parts, whereby $n$ is the divisor and the quotient is the size of such a part. This model could lead students to the intuitive rule that the quotient is smaller than the dividend, which leads them to consider that the result of a division always results in a smaller number (Bell et al., 1981; Fischbein et al., 1985; Greer, 1987; Hart et al., 1981). These assumptions, acquired and developed with the learning of natural numbers, are not always correct when working with rational numbers, since they depend on the specific rational numbers involved. For example, when you multiply by a rational number smaller than 1 , the result will be smaller (e.g. $4 \times 0.5$ or $4 \times 1 / 2)$.

In order to examine whether natural numbers properties are indeed being implicitly or explicitly applied in these cases, previous research has used tests that differentiated between congruent items (those in which natural number knowledge is compatible with rational number knowledge and leads to a correct answer) and incongruent items (those in which natural number knowledge is incompatible with rational number knowledge and leads to an incorrect answer) (Christou, 2015; Siegler \& Lortie-Forgues, 2015; Van Hoof et al., 2015a). In this way, significantly higher accuracy in congruent items compared to incongruent items is taken as evidence for the (inappropriate) use of natural number properties.

Van Hoof et al. (2015a) found that eighth-, tenth-, and twelfth-grade students were accurate in solving algebraic expressions when knowledge of natural numbers led them to the correct answer. For instance, in the congruent item "Can this expression be true? $m<4 \times m$ ”, students were more accurate since this expression is true for $m$ being any natural number. However, they had difficulties in expressions where natural number knowledge led them to an incorrect answer. For example, in the incongruent item "Can this expression be true? $3<3 \div$ ", if students use natural number knowledge, they 
would say that it cannot be true since $m$ must necessarily be a rational number smaller than one. These results have also been obtained with undergraduate students (Obersteiner et al., 2016).

In the same line, Christou (2015) observed that fifth- and sixth-grade students accurately solved items in which a missing number was a natural number or a rational number larger than one (congruent item, e.g. $8 \div \ldots=5 \rightarrow$ possible or impossible?). However, they had difficulties solving items in which a missing number was necessarily a rational number smaller than one (incongruent item, e.g. $\_\times 4=1 \rightarrow$ possible or impossible?). In order to correctly solve these items, they had to realise that multiplications can result in a smaller number and divisions can result in a larger number.

Fischbein et al. (1985) observed that fifth-, seventh-, and ninth-grade students chose the incorrect operation to solve word problems that involved multiplying a natural number by a decimal number smaller than one (incongruent item, e.g. The price of $1 \mathrm{~m}$ of suit fabric is 15,000 lire. What is the price of $0.65 \mathrm{~m}$ ?). Most of them considered that the result should be obtained by dividing $15000 \div 0.65$. The same occurred with divisions since they incorrectly solved word problems that involved dividing a natural number by a decimal number smaller than one (incongruent item, e.g. I spent 900 lire for 0.75 hg of cocoa. What is the price of 1 hg?). Most of them considered that the result should be obtained by multiplying $900 \times 0.75$. This research was only carried out with decimal numbers. Van Hoof et al. (2015b) included the fraction and decimal representation of rational numbers. In a study with fourth-, sixth-, eighth-, tenth-, and twelfth-grade students, these authors also found low accuracies in incongruent word problems with both fractions and decimal numbers. Furthermore, they found that both primary and secondary school students accurately estimated the result of a 
multiplication of a natural number by a rational number larger than one (congruent item, e.g. Is the outcome of $2 \times 1.5$ smaller or larger than 2?), and that they were inaccurate when the rational number was smaller than one (incongruent item, e.g. Is the outcome of $8 \times 1 / 2$ smaller or larger than $8 ?)$. Similarly, in division items, students correctly solved congruent items (e.g. Is the outcome of $5 \div 7 / 4$ smaller or larger than 5?) and incorrectly solved the incongruent ones (e.g. Is the outcome of $6 \div 0.3$ smaller or larger than 6?). Similar results have been found in undergraduate students (Siegler \& LortieForgues, 2015; Vamvakoussi et al., 2012), showing that these difficulties persist into adulthood. Regarding the mode of representation, no significant differences were found between students' accuracy in fractions and decimal numbers, concluding that the natural number bias was equally strong in both representations.

These results -higher accuracy in congruent items than in incongruent itemsunderline how students follow the implicit model "multiplication makes larger" and “division makes smaller" when estimating the result of multiplications and divisions with rational numbers.

\section{Objective of the present study}

The above-mentioned studies focused on students' average accuracy in arithmetic operations with rational numbers. They did not, however, study explicit individual differences among students depending on the nature of the operation, the mode of representation and the nature of the item. In this sense, we considered it valuable to extend previous research by determining primary and secondary school students' profiles according to their answer patterns in addition, subtraction, multiplication and division items, both with decimals and with fractions. Thereby, we use a variety of items that were used in the previous research as reviewed above. We are also interested in analysing the evolution and predominance of each profile from $5^{\text {th }}$ to $10^{\text {th }}$ grade. This 
allows examining possible trajectories of correct reasoning as well as detecting a large potential range of incorrect and qualitatively different ways students reason about rational arithmetic operations knowledge.

Our hypotheses underlying this objective is that there could be not just differences among students in their performances on the test items, but also qualitative differences among the students in the way they respond to particular items, depending on the nature of the operation, the mode of representation and the nature of the item. Furthermore, it is possible that other incorrect ways of reasoning different from the use of natural number knowledge may pop up in the study. The present cross-sectional study from $5^{\text {th }}$ to $10^{\text {th }}$ grade also could provide us information about the predominance of the different kinds of reasoning observed in each grade, and the evolution of these kinds of reasoning along grades.

\section{Method}

\section{Participants}

Participants were 1,262 Spanish primary and secondary school students distributed over $5^{\text {th }}$ grade (10-11 years old), $6^{\text {th }}$ grade (11-12 years old), $7^{\text {th }}$ grade (12-13 years old), $8^{\text {th }}$ grade (13-14 years old), $9^{\text {th }}$ grade (14-15 years old), and $10^{\text {th }}$ grade ( $15-16$ years old) (Table 1). There was approximately the same number of boys and girls in each age group. The participating schools were spread over nine cities (five primary schools and five secondary schools) and students were from mixed socio-economic backgrounds.

\section{TABLE 1 APPEARS HERE}

In the Spanish curriculum, the teaching of fractions and decimal numbers starts in $3^{\text {rd }}$ grade of primary education. In $5^{\text {th }}$ grade, students should: i) know the meaning of tenths, hundredths, and thousandths, being able to decompose and compose decimal 
numbers; ii) be able to represent fractions and decimals on the number line, comparing and ordering them and recognising their relationship; and iii) be able to conduct operations with them.

\section{Instrument and procedure}

The instrument consisted of an adaptation of the arithmetic operation items in the RNST (Rational Number Sense Test), as developed and validated by Van Hoof et al. (2015b). The RNST is a test with 177 items related to the three domains of the natural number bias (arithmetic operations, density and size). Van Hoof et al. (2015b) validated and used 63 out of these 177 items, which comprises items from the three domains: 15 items of arithmetic operations, 15 items of density, and 33 items of size. Our paper-and-pencil test consisted of 16 arithmetic operation items.

As our objective was to examine individual differences among students depending on the nature of the operation, the mode of representation and the nature of the item, the selected items used in our instrument presented a range of different characteristics. Regarding the nature of the operation, there were addition, subtraction, multiplication and division items. With regard to the mode of representation, there were fraction and decimal number items. Concerning the nature of the item, we used items where students had to anticipate the size of the result of an operation (question items) and items where they had to anticipate the operation that leads to the solution of a word problem (word problem items). As we mentioned in our objectives and hypotheses, this variety of characteristics allowed us to examine differences in students' performances depending on the nature of the operation, the mode of representation and the nature of the item, and gave us the opportunity to examine if students used other ways of 
reasoning different from the use of natural number knowledge. Following, we explain in detail the structure of the test and the characteristics of the 16 items used.

There were four addition and subtraction items: an addition with fractions, an addition with decimal numbers, a subtraction with fractions and a subtraction with decimal numbers (Table 2). All these items were considered incongruent since the procedures of addition and subtraction with natural numbers cannot be applied to rational numbers. The kind of natural number bias error that may be observed in addition and subtraction items is a procedural one: calculating the result by applying procedures that only hold for natural numbers. Therefore, for the addition and subtraction items we did not include word problems.

\section{TABLE 2 APPEARS HERE}

Furthermore, there were 12 multiplication and division items: eight question items and four word problem (WP) items. The objective was to analyse students' performances in both types of items (questions and word problems used in previous literature). Regarding question items (Table 3), four were congruent (a multiplication with fractions, a multiplication with decimal numbers, a division with fractions and a division with decimal numbers) and four were incongruent (a multiplication with fractions, a multiplication with decimal numbers, a division with fractions and a division with decimal numbers). In the congruent items, if students used a reasoning based on the natural number bias "multiplication always makes larger" and "division always makes smaller", they would give a correct answer. If students used the same reasoning in incongruent items, they would provide an incorrect answer. To avoid too many items for the youngest students, we only included four incongruent word problem items (two multiplication and two division items with fractions and decimal numbers) 
(Table 4) that gave us the necessary information to analyse if students were also

following the intuitive rules (NNB) in multiplication and division word problem items.

TABLE 3 APPEARS HERE

\section{TABLE 4 APPEARS HERE}

Eight different versions of the test were designed presenting the 16 items in a randomised order to avoid that the order of the items could affect the results. Students were asked to individually solve the paper-and-pencil test during a mathematics lesson at school. The data were collected during the spring of 2018. Participants (and schools) were recruited randomly and parental consent was obtained for all of them. No time limit was used, as a time limitation could encourage natural number biased reasoning (Vamvakoussi et al., 2012).

\section{Analysis}

The students' answers to the 16 items were categorized individually using the following categories (we used the term "Rest" to refer to the answers that were neither the correct answer nor the NNB answer):

- In the congruent multiplication question items, the answers were categorized as:

i) Correct: students who correctly marked "larger"; ii) Rest: students who marked "smaller"; and iii) Blank answers. In the congruent division question items, the answers were categorized as: i) Correct: students who correctly marked "smaller"; ii) Rest: students who marked "larger"; and iii) Blank answers.

- In the incongruent multiplication question items, the answers were categorized as: i) Correct: students who correctly marked "smaller"; ii) NNB (natural number bias): students who marked "larger"; and iii) Blank answers. In the 
incongruent division question items, the answers were categorized as: i) Correct: students who correctly marked "larger"; ii) NNB (natural number bias): students who marked "smaller"; and iii) Blank answers.

- In multiplication word problem items, the answers were categorized as: i) Correct: students who correctly marked the multiplication option; ii) $N N B$ : students who marked the division with the dividend larger than the divisor; iii) Rest: students who marked the division with the dividend smaller than the divisor; and iv) Blank answers. In division word problem items, the answers were categorized as: i) Correct: students who marked the correct division option; ii) $N N B$ : students who marked the multiplication option; iii) Rest: students who marked the division with the dividend smaller than the divisor; and iv) Blank answers.

- For the addition and subtraction items, the answers were categorized as: i) Correct: students who correctly calculated additions/subtractions; ii) NNB: students who added/subtracted numerators and denominator separately in fraction items; added/subtracted decimals without taking into account the place value in decimal items. For example, $2 / 6+1 / 3$ is $3 / 9$ or $0.36-0.2$ is 0.34 ; iii) Rest: students who did other mistakes in additions/subtractions; and iv) Blank answers.

With these 16 categorical variables (the answer to each item), a TwoStep Cluster Analysis (in SPSS 25) with categorical data was performed, in order to find groups of students (profiles) with qualitatively similar response patterns (same answers to the same items). This analysis, developed by Chiu, Fang, Chen, Wang, and Jeris (2001), copes effectively with very large datasets. The algorithm is based on a two-stage 
approach. In the first stage, the algorithm undertakes a procedure that is very similar to the k-means algorithm. Based on these results, during the second stage, the procedure follows a modified hierarchical agglomerative clustering process that combines the objects sequentially so as to form homogenous clusters. Moreover, the procedure guides decision-making regarding the number of clusters to retain from the data, by calculating measures of fit such as the Akaike Information Criterion (AIC) or Bayesian Information Criterion (BIC) (Sarstedt \& Mooi, 2014).

\section{Results}

The results of the study are divided into the three sections below: first, we determine the number of profiles; second, we describe the different profiles obtained; and finally, we examine the evolution of these profiles from $5^{\text {th }}$ grade of primary school to the end of secondary education $\left(10^{\text {th }}\right.$ grade).

\section{Determining the number of profiles}

The number of profiles was based on a low value of BIC (Table 5) as well as an interpretative viewpoint (see below). The BIC value indicates an optimal balance between the model's fit and the model's complexity in terms of the number of estimated parameters $(=\mathrm{fp})$. Schwarz (1978) proposed the BIC criterion, which takes the sample size into account. This value is calculated as follows: $\mathrm{BIC}=-2 \log L+\log (I) \times \mathrm{fp}$ where $L$ is the likelihood of the model and $I$ the sample size. Due to the large sample size in our study, we chose this value.

\section{TABLE 5 APPEARS HERE}

The eight-state model was preferred from an interpretation point of view since it was more theoretically interesting. As shown in Figure 1, which displays the frequency percentages of the most widely used categories in all the items, the additional profile 
(Cluster $8, n=160$, eight-class solution) that appeared in the eight-class solution compared to the seven-class solution (Cluster 3, $n=274$, seven-class solution) is of theoretical interest. It allows us to differentiate between students who, in addition and subtraction items, correctly solved decimal items (mean Correct answer $=85.27 \%$ ) but incorrectly answered fractions items, making mistakes different than the natural number bias (mean Rest answer $=72.26 \%)($ Cluster 3, $n=146$, eight-class solution $)$; and students who solved fraction and decimal addition and subtraction items correctly $($ mean Correct answer $=92.97 \%)($ Cluster $8, n=160$, eight-class solution $)$.

\section{FIGURE 1 APPREARS HERE}

In contrast, as Figure 2 shows, the additional profile that appeared in the nineclass solution (Cluster 4, $n=181$, nine-class solution), does not present much theoretical interest compared to the eight-class solution (Cluster 7, $n=326$, eight-class solution). It only distinguishes between a group of learners with a general high performance, but who answered "larger" in the division $37 \div 1.2$ (mean Rest answer $=$ $66.0 \%$ ) and based their answers in the fraction multiplication word problem on natural number knowledge (mean $N N B$ answer $=80.0 \%)($ Cluster $2, n=200$, nine-class solution), and a group of learners with a general high performance (as Cluster 7 in the eight-class solution) in all the items (Cluster $4, n=181$, nine-class solution).

FIGURE 2 APPEARS HERE

\section{Characteristics of the profiles identified}

Figure 3 shows the characteristics of the profiles identified. The X-axis consists of the 16 test items in the following order: the four congruent question items, the four incongruent question items, the four incongruent word problem items, and the four addition and subtraction items. The Y-axis consists of the frequency percentages of the most widely used categories. Below, we describe the characteristics of each of the eight 
profiles and indicate the percentage of students in each profile.

- Almost correct $(25.8 \% ; n=326)$ : students of this cluster solved the majority of the items correctly (mean Correct answer $=69.33 \%$ ). These students were more accurate in addition and subtraction items (mean Correct answer $=92.79 \%$ ) than in division and multiplication items (mean Correct answer $=61.50 \%$ ). A logistic regression analysis showed that this difference was significant $(p<0.001)$. However, there was a group of students who were still natural number knowledge biased, particularly in multiplication and division word problems items (mean $N N B$ answer $=32.75 \%$ ) and division question items (mean $N N B$ answer $=55.37 \%$ ). Furthermore, some students solved congruent items using "a reverse thinking", in other words, students marked the option "smaller" in multiplications items and the option "larger" in divisions items (mean Rest answer: $32.13 \%$ ). The use of reverse thinking was bigger in division items $($ mean Rest answer $=42.64 \%)$ than in multiplication items (mean Rest answer $=$ $21.63 \%)$. This difference $(42.64 \%$ vs. $21.63 \%)$ was significant $(p<0.001)$.

- Full NNB $(10.6 \% ; n=134)$ : students of this cluster incorrectly solved the majority of the incongruent multiplication and division items based on natural number knowledge (mean $N N B$ answer $=60.45 \%)$ and used natural number knowledge in addition and subtraction items (mean $N N B$ answer $=79.48 \%$ ) both in fractions and decimals. They were more natural number knowledge biased in question items (mean $N N B$ answer $=75.75 \%$ ) than in word problem items (mean $N N B$ answer $=45.15 \%)$. This difference was significant $(p<0.001)$. In contrast, they correctly solved congruent multiplication and division items (mean Correct answer $=74.81 \%)$. 
- Multiplication/division and fraction addition/subtraction NNB $(11.6 \% ; n=146)$ : students of this cluster correctly solved congruent multiplication and division items (mean Correct answer $=97.09 \%$ ), but they incorrectly solved incongruent multiplication and division items using natural number knowledge (mean $N N B$ answer $=67.38 \%$ ). Furthermore, students were more natural number knowledge biased in question items (mean $N N B$ answer $=90.58 \%$ ) than in word problem items (mean $N N B$ answer $=44.18 \%)$. This difference was significant $(p<$ 0.001). In addition and subtraction items, they solved decimal items correctly (mean Correct answer 77.74\%), but fraction items incorrectly using natural number knowledge (mean $N N B$ answer $=90.41 \%)$.

- Multiplication/division NNB and blank fraction addition/subtraction answers $(9.6 \% ; n=121):$ students of this cluster correctly solved congruent multiplication and division items (mean Correct answer $=78.72 \%$ ), but incorrectly solved incongruent multiplication and division items using natural number knowledge (mean $N N B$ answer $=58.37 \%$ ). Furthermore, they were more natural number knowledge biased in question items (mean $N N B$ answer $=$ $73.55 \%$ ) than in word problem items (mean $N N B$ answer $=43.18 \%$ ). This difference was significant $(p<0.001)$. In addition and subtraction items, students solved decimal items accurately (mean Correct answer $=59.09 \%$ ). However, in fraction items, these students provided a large number of blank answers (mean Blank answer $=82.06 \%)$.

- Multiplication/division NNB and low fraction addition/subtraction performance $(11.6 \% ; n=146)$ : students of this cluster correctly solved congruent multiplication and division items (mean Correct answer $=86.82 \%$ ), but 
incorrectly solved incongruent multiplication and division items using natural number knowledge (mean $N N B$ answer $=63.53 \%$ ). Furthermore, they were more natural number knowledge biased in question items (mean $N N B$ answer $=$ $82.19 \%$ ) than in word problem items (mean $N N B$ answer $=44.86 \%$ ). This difference was significant $(p<0.001)$. In addition and subtraction items, students solved decimal items accurately (mean Correct answer $=85.27 \%$ ). However, in fraction items students made other mistakes (mean Rest answer $=$ $72.26 \%)$.

- Multiplication/division NNB $(12.7 \% ; n=160)$ : students of this cluster solved fraction and decimal addition and subtraction items correctly (mean Correct answer $=92.97 \%)$. However, in multiplication and division items, they correctly solved congruent items (mean Correct answer $=92.5 \%$ ), but incorrectly solved incongruent ones using natural number knowledge (mean $N N B$ answer $=$ $66.88 \%$ ). Furthermore, students were more natural number knowledge biased in question items (mean $N N B$ answer $=85.94 \%$ ) than in word problem items (mean $N N B$ answer $=47.81 \%)$. This difference was significant $(p<0.001)$.

- Multiplication/division reverse thinking and fraction addition/subtraction NNB $(13.6 \% ; n=172)$ : students of this cluster were more accurate in congruent multiplication and division items (mean Correct answer $=56.10 \%$ ), than in incongruent ones (mean Correct answer $=42.37 \%$ ). This difference was significant $(p<0.001)$. There was a subgroup of students who used natural number knowledge in incongruent items (mean $N N B$ answer $=44.84 \%$ ). Moreover, some students solved congruent items using a "a reverse thinking", in other words, students who marked the option "smaller" in multiplications items 
and the option "larger" in divisions items (category of Rest, mean: 43.61\%). The use of reverse thinking was bigger in division items (mean Rest answer $=$ $51.45 \%$ ) than in multiplication items (mean Rest answer $=35.76 \%$ ). The statistical analysis showed that this difference was significant $(p<0.001)$. In addition and subtraction items, they solved decimal items correctly (mean Correct answer $=76.74 \%$ ), but fraction items incorrectly using natural number knowledge $($ mean $N N B$ answer $=68.02 \%)$.

- Remainder $(4.5 \% ; n=57)$ : students of this cluster had a generally low performance (mean Correct answer $=22.04 \%$ ), and solved the items without any identifiable pattern.

\section{FIGURE 3 APPEARS HERE}

\section{Evolution of the profiles by grade}

In terms of evolution (Figure 4), the percentage of students in the Almost correct profile increased as the grades advanced $\left(0.49 \%\right.$ in $5^{\text {th }}$ grade and $51.90 \%$ in $10^{\text {th }}$ grade $)$. A logistic regression analysis showed a significant main effect of grade $\chi^{2}(5, N=1262)=$ $140.126, p<0.001$. Nevertheless, by $10^{\text {th }}$ grade, approximately a half of students were still not included in this profile. The percentages of the profiles Full NNB and Multiplication/division and fraction addition/subtraction $N N B$ were higher in the first years, and almost disappeared by the end of secondary school (from $23.41 \%$ in $5^{\text {th }}$ grade to $1.90 \%$ in $10^{\text {th }}$ grade in the Full $N N B$ profile and from $26.34 \%$ in $5^{\text {th }}$ grade to $3.81 \%$ in $10^{\text {th }}$ grade in the Multiplication/division and fraction addition/subtraction NNB profile). The statistical analysis also showed a significant grade effect for the two groups $\chi^{2}(5, N$ $=1262)=60.867, p<0.001$ and $\chi^{2}(5, N=1262)=53.472, p<0.001$, respectively.

The percentage of the profile Multiplication/Division NNB and blank fraction addition/subtraction answer increased from $5^{\text {th }}$ to $6^{\text {th }}$ grade $\left(10.73 \%\right.$ in $5^{\text {th }}$ grade and 
$14.16 \%$ in $6^{\text {th }}$ grade), and then decreased as grades advanced but did not disappear by the end of secondary school (5.24\% in $10^{\text {th }}$ grade). The statistical analysis showed a significant grade effect $\chi^{2}(5, N=1262)=14.883, p=0.011$. However, the percentage of the profile Multiplication/division NNB and low fraction addition/subtraction performance also increased from $5^{\text {th }}$ to $6^{\text {th }}$ grade $\left(7.80 \%\right.$ in $5^{\text {th }}$ grade and $15.98 \%$ in $6^{\text {th }}$ grade), but then remained stable by the end of secondary school (around 10\%). The logistic regression did not show a significant grade effect $\chi^{2}(5, N=1262)=8.928, p=$ 0.112 .

\section{FIGURE 4 APPEARS HERE}

The percentage of students in the Multiplication/division NNB profile increased from $5^{\text {th }}$ to $10^{\text {th }}$ grade (from $0.98 \%$ in $5^{\text {th }}$ grade to $17.62 \%$ in $10^{\text {th }}$ grade) and the logistic regression analysis showed a significant grade effect $\chi^{2}(5, N=1262)=20.087, p<$ 0.001 . This result shows that around $20 \%$ of tenth-grade students were able to add and subtract fractions and decimals correctly, but still were natural number biased in multiplications and divisions. Regarding the Multiplication/division reverse thinking and fraction addition/subtraction $N N B$ profile, it was stable from $5^{\text {th }}$ to $7^{\text {th }}$ grade (around $20 \%)$ and then, there was a decrease $\left(9.52 \%\right.$ in $10^{\text {th }}$ grade). The logistic regression analysis showed also a significant grade effect in this group $\chi^{2}(5, N=1262)$ $=12.760, p=0.026$. Finally, the percentage of Remainder profile was small throughout all grades, and almost disappeared in the last years $\left(13.17 \%\right.$ in $5^{\text {th }}$ grade and $0.95 \%$ in $10^{\text {th }}$ grade).

These results seem to indicate, on the one hand, that the tendency to rely on natural number properties is not overcome at the same time in additions and subtractions as in multiplications and divisions. This result is evidenced by the decrease along grades of Full NNB, Multiplication/division and fraction addition/subtraction 
NNB, Multiplication/division NNB and blank fraction addition/subtraction answers, Multiplication/division NNB and low fraction addition/subtraction performance profiles and the increase of the Multiplication/division $N N B$ profile. In this sense, students first tend to apply natural number knowledge less often in addition and subtraction items, and only later in multiplications and divisions. The application of natural number properties in these latter operations seems to persist until the end of secondary school. On the other hand, differences among additions and subtractions were not identified. However, although the natural number bias was predominantly equally strong both in multiplications and divisions in the majority of the profiles, Almost correct and Multiplication/division reverse thinking and fraction addition/subtraction NNB profiles showed the existence of a "reverse thinking". Moreover, the evolution shows how the Almost correct profile increased as grades advanced, and that the Multiplication/division reverse thinking and fraction addition/subtraction $N N B$ remained stable by the end of secondary school.

Finally, results have also shown differences regarding representation only in addition and subtraction items. The profile Multiplication/division and fraction addition/subtraction $N N B$ shows the existence of students who no longer apply natural number properties in additions and subtractions in decimal numbers, but persist doing so in fractions. Whereas in multiplication and division items, all profiles showed that the natural number bias is equally strong both for fractions and decimals. However, differences regarding the type of item have been found in multiplication and division items. In the majority of the profiles, students were more natural number knowledge biased in question items than in word problem items. In other words, they were more biased when they had to anticipate the result than when they had to anticipate the operation in a word problem. 


\section{Discussion and conclusions}

In the present study, we presented results from a cross-sectional study with a large sample of students from the last grades of primary school to the last grades of secondary school. Using a TwoStep Cluster Analysis, we found different profiles, that is, different students' reasoning when solving rational number operations. These profiles can help us to identify differences in understanding - or misunderstanding- of arithmetic operations with rational numbers that depends on the type of arithmetic operation (addition, subtraction, multiplication or division), the representation (fraction or decimal number), or the nature of the item (question/word problem).

Results showed differences in the tendency to apply natural number properties beyond their applicability range depending on the type of arithmetic operation. The evolution showed that the Full NNB decreased and seemed to disappear by the end of secondary school, meanwhile the Multiplication/division NNB (students who were natural number biased only in multiplication and division) increased as grades advanced. This result suggests that the natural number bias is first overcome in addition and subtraction, and later in multiplication and division. This result is in line with previous research that has shown higher levels of success in additions and subtractions than in multiplications and divisions, pointing out that the natural number bias is stronger in multiplications and divisions (Siegler \& Lortie-Forgues, 2015; Van Hoof et al., 2015a).

Moreover, results showed that there were no differences in students' answers between addition and subtraction. However, some differences were found in students' answers between multiplication and division. This issue was pointed out in Van Hoof et al.'s (2015b) study, who found that secondary school students were more natural number biased in divisions than in multiplications. However, Christou (2015) showed 
the opposite with primary school students. Although our results generally show that the tendency to apply natural number properties is equally strong in division as in multiplication, since the majority of the profiles considered that multiplications make larger and divisions make smaller, the existence of the Almost correct and Multiplication/division reverse thinking and fraction addition/subtraction NNB profiles indicate that for some students, difficulties in division and multiplication items are not related to the natural number bias as such. These students considered that divisions make larger and multiplications make smaller when a rational number is involved, even when the rational number is larger than one. This "reverse thinking" was stronger in division items than in multiplication items. Furthermore, the evolution showed that the Almost correct profile increased as grades advanced, and the Multiplication/division reverse thinking and fraction addition/subtraction $N N B$ remained stable by the end of secondary school. Therefore, the evolution of the profiles seems to indicate that when the tendency to apply natural number properties decreased, it can be followed by a qualitatively different error (reverse thinking) before reaching the stage of correct understanding, and this error is stronger in divisions than in multiplications.

The inclusion of items with the same characteristics both in decimals and fractions allowed us to identify differences in students' performances depending on the representation. As can be observed in the profiles Multiplication/division and fraction addition/subtraction NNB, Multiplication/division NNB and blank fraction addition/subtraction answers, Multiplication/division NNB and low fraction addition/subtraction performance, and Multiplication/division reverse thinking and fraction addition/subtraction $N N B$, students correctly answered decimal addition and subtraction items, but incorrectly answered the variants with fractions. These results show that the natural number bias in students' understanding of addition and subtraction 
is stronger in fractions. However, there are no differences between multiplication and division items regarding representation. This result is in line with previous studies that had not found differences in the natural number bias between fractions and decimal numbers (Fischbein et al., 1985; Siegler \& Lortie-Forgues, 2015; Van Hoof et al., 2015b), in our case, only for multiplications and divisions.

Finally, some differences have also been found depending on the nature of the item. The majority of the profiles answered more in a natural number biased way in question items than in word problem items. In other words, for a subgroup of students, anticipating the result of a numerical expression was more affected by natural number knowledge than anticipating the operation to be applied in a word problem. Therefore, our research extends previous research (Van Hoof et al., 2015b) showing that some students correctly choose the operation for solving a word problem when the same kind of rational number is involved (accepting that multiplications can make smaller and divisions can make larger), but incorrectly anticipate the result of a multiplication/division when a rational number smaller than one is involved. This result seems to show that those items where students have to anticipate the result (question items) can favour the misunderstanding of multiplication/division with rational numbers in the same manner as with natural numbers than those items where students have to anticipate the operation in a word problem (word problem items). A possible explanation is that the context of the problem might be helpful in choosing the correct operation and could help students not to apply the properties of natural numbers.

Regarding the evolution of the profiles by grade, although generally speaking the tendency to apply natural number properties decreased along grades, it did not disappear in the last grades of secondary school, regarding neither fractions nor decimal numbers in multiplications and divisions. Therefore, a large subgroup of students by the 
end of secondary school still consider that multiplications always make larger and divisions always make smaller. In fact, although the percentage of the Almost correct profile increased, it was only reached by around $50 \%$ of tenth-grade students.

Our results may raise reflections on how to handle this complex phenomenon in education. Firstly, according to the results, the tendency to apply natural number properties does not seem to disappear towards the end of secondary education. It does for some students, but one subgroup is still clearly showing this tendency. Other students seem to end up with only a partial understanding, while still being affected to some extent. We can thus consider that not only primary school teachers but also secondary school teachers should be aware of this tendency and of the fact that it may manifest itself in various intermediate forms.

Secondly, as the tendency to apply natural number properties has generally been more frequently observed in multiplication and division items, a stronger awareness of the possible negative consequences of introducing multiplication through the repeated addition model and introducing division through the equal sharing model may help to prevent students' later conceptual difficulties with these operations (Van Hoof et al., 2015a).

Our study has also theoretical implications, particularly, the profiles of students' arithmetic operations with rational numbers understanding identified. However, we can highlight also some limitations. We are aware that a cross-sectional study cannot show a real evolution of each reasoning along grades. In this sense, future research would benefit from longitudinal designs (within the same age range) to examine how learners' individual understanding of arithmetic operations with rational numbers progresses over time (see for example, McMullen \& Van Hoof, 2020). A particularly promising perspective in this respect is to look at the phenomenon from the reorganization 
perspective (Biddlecomb, 2002; Olive, 1999; Steffe, 2002), which focuses less on the dissimilarities between learners' prior knowledge and the to-be-acquired insight, but rather considers learners' prior knowledge as productive and essential in the evolution towards a correct understanding. The various profiles that we identified in our study might indeed be the result of learners' meaningful attempts to reorganize their prior knowledge in light of new information.

Furthermore, although our results evidenced different students' profiles (ways of reasoning) related to their understanding of rational number operations, these ways of thinking are inferred from answer patterns obtained in a quantitative test. A qualitative perspective, such as those used in the reorganization perspective mentioned before, would also be valuable to support and build a deeper understanding of the profiles we identified. It would be worth conducting interviews with students belonging to the different profiles not only to support our results, but also to gain clearer insights into students' different ways of reasoning. This kind of research could also lead to explaining the differences observed in our study regarding the items in which students had to anticipate the result of a multiplication or a division (question items), and the items in which they had to anticipate an operation in a word problem (word problem items). It could also contribute to advance our comprehension of students' use of "reverse thinking", especially in division items. As mentioned previously, these difficulties are not directly related to the natural number bias, but they have something to do with natural number bias. That is, the learners now overgeneralise their newly acquired insight about arithmetic operations in the opposite. Therefore, they "unlearn" the natural number bias for a kind of "rational number bias" considering that when a rational number is involved, division always makes larger. 


\section{Acknowledgements}

This research was carried out with the support of the project PROMETEO/2017/135 from Conselleria d'Educació, Investigació, Cultura i Esport (Generalitat Valenciana, Spain), and the predoctoral grant from the University of Alicante (UAFPU2018-035), and the postdoctoral grant (I-PI 69-20).

\section{References}

Behr, M. J., Lesh, R., Post, T., \& Silver E. (1983). Rational Number Concepts. In R. Lesh, \& M. Landau (Eds.), Acquisition of Mathematics Concepts and Processes, (pp. 91-125). New York, NY, USA: Academic Press.

Behr, M., Wachsmuth, I., Post T., \& Lesh R. (1984). Order and equivalence of rational numbers: A clinical teaching experiment. Journal for Research in Mathematics Education, 15(5), 323-341.

Bell, A., Swan, M., \& Taylor, G. (1981). Choice of operation in verbal problems with decimal numbers. Educational Studies in Mathematics, 12(4), 399-420. https://doi.org/10.1007/BF00308139

Biddlecomb, B. D. (2002). Numerical knowledge as enabling and constraining fraction knowledge: An example of the reorganization hypothesis. The Journal of Mathematical Behavior, 21(2), 167-190. https://doi.org/10.1016/S0732$\underline{3123(02) 00117-7}$

Chiu, T., Fang, D., Chen, J., Wang, Y., \& Jeris, C. (2001). A robust and scalable clustering algorithm for mixed type attributes in large database environment. In Proceedings of the seventh ACM SIGKDD International Conference on Knowledge Discovery and Data Mining (pp. 263-268). ACM. https://doi.org/10.1145/502512.502549

Christou, K. P. (2015). Natural number bias in operations with missing numbers. ZDM Mathematics Education, 47(5), 747-758. https://doi.org/10.1007/s11858-015$\underline{0675-6}$ 
Cramer, K., Post, T., \& delMas, R. (2002). Initial fraction learning by fourth- and fifthgrade students: A comparison of the effects of using commercial curricula with the effects of using the rational number project curriculum. Journal for Research in Mathematics Education, 33(2), 111-144. https://doi.org/10.2307/749646

Fischbein, E., Deri, M., Nello, M. S., \& Marino, M. S. (1985). The role of implicit models in solving verbal problems in multiplication and division. Journal for Research in Mathematics Education, 16(1), 3-17. https://doi.org/10.2307/748969

Gómez, D., \& Dartnell, P. (2019). Middle schoolers' biases and strategies in a fraction comparison task. International Journal of Science and Mathematics Education, 17(6), 1233-1250. https://doi.org/10.1007/s10763-018-9913-z

González-Forte, J. M., Fernández, C., Van Hoof, J., \& Van Dooren, W. (2020). Various ways to determine rational number size: an exploration across primary and secondary education. European Journal of Psychology of Education, 35, 549565. https://doi.org/10.1007/s10212-019-00440-w

Greer, B. (1987). Nonconservation of multiplication and division involving decimals. Journal for Research in Mathematics Education, 18(1), 37-45. https://doi.org/10.2307/749535

Hart, K. M., Brown, M. L., Kuchemann, D. E., Kerslake, D., Ruddock, G., \& McCartney, M. (1981). Children's understanding of mathematics: 11-16. London, United Kingdom: John Murray.

Hiebert, J., \& Wearne, D. (1985). A model of students' decimal computation procedures. Cognition and Instruction, 2(3-4), 175-205. https://doi.org/10.1080/07370008.1985.9648916 
Kerslake, D. (1986). Fractions: Children's strategies and errors. A report of the strategies and errors in secondary mathematics project. Windsor, United Kingdom: NFER-NELSON.

Kieren, T. E. (1993). Rational and fractional numbers: From quotient fields to recursive understanding. In T. P. Carpenter, E. Fennema, \& T. A. Romberg (Eds.), Rational numbers: An integration of research (pp. 49-84). Hillsdale, NJ: Lawrence Erlbaum Associates, Inc.

Lortie-Forgues, H., Tian, J., \& Siegler, R. S. (2015). Why is learning fraction and decimal arithmetic so difficult? Developmental Review, 38, 201-221. https://doi.org/10.1016/j.dr.2015.07.008

McMullen, J., \& Van Hoof, J. (2020). The role of rational number density knowledge in mathematical development. Learning and Instruction, 65 https://doi:10.1016/j./learninstruc.2019.101228

McMullen, J., Laakkonen, E., Hannula-Sormunen, M., \& Lehtinen, E. (2015). Modeling the developmental trajectories of rational number concept(s). Learning and Instruction, 37, 14-20. https://doi.org/10.1016/j.learninstruc.2013.12.004

Merenluoto, K., \& Lehtinen, E. (2002). Conceptual change in mathematics: Understanding the real numbers. In M. Limon \& L. Mason (Eds.), Reconsidering Conceptual Change: Issues in Theory and Practice (pp. 233-258). Dordrecht, The Netherlands: Kluwer.

Moss, J. (2005). Pipes, tubs, and beakers: New approaches to teaching the rationalnumber system. In M. S. Donovan \& J. D. Bransford(Eds.), How students learn: History, math, and science in the classroom (pp. 309-349). Washington, DC: National Academies Press. 
Namkung, J. M., Fuchs, L. S., \& Koziol, N. (2018). Does initial learning about the meaning of fractions present similar challenges for students with and without adequate whole-number skill? Learning and individual differences, 61, 151-157. https://doi.org/10.1016/j.lindif.2017.11.018

Ni, Y., \& Zhou, Y. D. (2005). Teaching and learning fraction and rational numbers: The origins and implications of whole number bias. Educational Psychologist, 40(1), 27-52. https://doi.org/10.1207/s15326985ep4001_3

Obersteiner, A., Van Hoof, J., Verschaffel, L., \& Van Dooren, W. (2016). Who can escape the natural number bias in rational number tasks? A study involving students and experts. British Journal of Psychology, 107, 537-555. https://doi.org/10.1111/bjop.12161

Olive, J. (1999). From fractions to rational numbers of arithmetic: A reorganization hypothesis. Mathematical Thinking and Learning, 1(4), 279-314. https://doi.org/10.1207/s15327833mt10104_2

Post, T. R. (1981). Fractions: Results and implications from National Assessment. Arithmetic Teacher, 28(9), 26-31.

Resnick, L. B., Nesher, P., Leonard, F., Magone, M., Omanson, S., \& Peled, I. (1989). Conceptual bases of arithmetic errors: The case of decimal fractions. Journal for Research in Mathematics Education, 20(1), 8-27. https://doi.org/10.2307/749095

Sarstedt, M., \& Mooi, E. (2014). A concise guide to market research. The process, data, and methods using IBM SPSS Statistics. Berlin, Germany: Springer.

Schwarz, G. (1978). Estimating the dimension of a model. The Annals of Statistics, 6, $461-464$. 
Siegler, R. S., \& Lortie-Forgues, H. (2015). Conceptual knowledge of fraction arithmetic. Journal of Educational Psychology, 107(3), 909-918. https://doi.org/10.1037/edu0000025

Siegler, R. S., \& Pyke, A. A. (2013). Developmental and individual differences in understanding of fractions. Developmental Psychology, 49(10), 1994-2004. https://doi.org/10.1037/a0031200

Siegler, R. S., Thompson, C. A., \& Schneider, M. (2011). An integrated theory of whole number and fractions development. Cognitive Psychology, 62(4), 273-296. https://doi.org/10.1016/j.cogpsych.2011.03.001

Smith, C. L., Solomon, G. E., \& Carey, S. (2005). Never getting to zero: Elementary school students' understanding of the infinite divisibility of number and matter. Cognitive Psychology, 51(2), 101-140. https://doi.org/10.1016/j.cogpsych.2005.03.001

Stafylidou, S., \& Vosniadou, S. (2004). The development of students' understanding of the numerical value of fractions. Learning and Instruction, 14(5), 503-518. https://doi.org/10.1016/j.learninstruc.2004.06.015

Steffe, L. P. (2002). A new hypothesis concerning children's fractional knowledge. The Journal of Mathematical Behavior, 20(3), 267-307. https://doi.org/10.1016/S0732-3123(02)00075-5

Streefland, L. (1991). Fractions in realistic mathematics education: A paradigm of developmental research. Dordrecht, The Netherlands: Kluwer.

Vamvakoussi, X., \& Vosniadou, S. (2004). Understanding the structure of the set of rational numbers: A conceptual change approach. Learning and Instruction, 14(5), 453-467. https://doi.org/10.1016/j.learninstruc.2004.06.013 
Vamvakoussi, X., Christou, K. P., \& Vosniadou, S. (2018). Bridging psychological and educational research on rational number knowledge. Journal of Numerical Cognition, 4(1), 84-106. https://doi.org/10.5964/jnc.v4i1.82

Vamvakoussi, X., Van Dooren, W., \& Verschaffel, L. (2012). Naturally biased? In search for reaction time evidence for a natural number bias in adults. The Journal of Mathematical Behavior, 31(3),

344-355. https://doi.org/10.1016/j.jmathb.2012.02.001

Van Dooren, W., Lehtinen, E., \& Verschaffel, L. (2015). Unraveling the gap between natural and rational numbers. Learning and Instruction, 37, 1-4. https://doi.org/10.1016/j.learninstruc.2015.01.001

Van Hoof, J., Degrande, T., Ceulemans, E., Verschaffel, L., \& Van Dooren, W. (2018). Towards a mathematically more correct understanding of rational numbers: A longitudinal study with upper elementary school learners. Learning and Individual Differences, 61, 99-108. https://doi.org/10.1016/j.lindif.2017.11.010

Van Hoof, J., Vandewalle, J., Verschaffel, L., \& Van Dooren, W. (2015a). In search for the natural number bias in secondary school students' interpretation of the effect of arithmetical operations. Learning and Instruction, 37, 30-38. https://doi.org/10.1016/j.learninstruc.2014.03.004

Van Hoof, J., Verschaffel, L., \& Van Dooren, W. (2015b). Inappropriately applying natural number properties in rational number tasks: Characterizing the development of the natural number bias through primary and secondary education. Educational Studies in Mathematics, 90(1), 39-56. https://doi.org/10.1007/s10649-015-9613-3 